\title{
First report of molecular detection of fluoroquinolone resistance-associated gyrA mutations in multidrug-resistant clinical Mycobacterium tuberculosis isolates in Kuwait
}

\author{
Noura M Al-Mutairi, Suhail Ahmad ${ }^{*}$ and Eiman Mokaddas
}

\begin{abstract}
Background: Nearly 5\% of all Mycobacterium tuberculosis strains worldwide are resistant at least to rifampicin and isoniazid (multidrug-resistant tuberculosis, MDR-TB). Inclusion of a fluoroquinolone and an injectable agent (kanamycin, amikacin or capreomycin) in multidrug therapy is crucial for proper treatment of MDR-TB. The incidence of MDR-TB in Kuwait is 1\%. MDR-TB strains additionally resistant to fluoroquinolones and injectable agents are defined as extensively drug-resistant (XDR-TB) strains and have been detected in $>55$ countries. Infections with XDR-TB strains have very poor prognosis. This study detected the occurrence of gyrA mutations associated with fluoroquinolone resistance among MDR-TB strains in Kuwait.

Findings: Direct DNA sequencing of quinolone resistance-determining region of gyrA gene was performed to detect fluoroquinolone resistance-associated mutations in 85 MDR-TB strains isolated from 55 TB patients and 25 pansusceptible M. tuberculosis strains. For isolates exhibiting gyrA mutations, 3'-end of rrs (16S rRNA) was sequenced for the detection of XDR-TB. Fingerprinting of fluoroquinolone resistant MDR-TB strains was performed by detecting mutations in three ( 81 bp hot-spot, N-terminal and cluster II) regions of rpoB, katG codon 315 and inhA-regulatory region, polymorphisms at gyrA codon 95 and kat $G$ codon 463 by DNA sequencing and by doublerepetitive-element PCR for determining strain relatedness. None of the pansusceptible but six of 85 MDR-TB strains contained gyrA mutations. Only gyrA codon 94 was mutated in all six (D94A in one and D94G in five) strains. Three of six mutant strains were recovered from the same patient while three other strains represented individual patient isolates. Fingerprinting studies identified all individual patient isolates as epidemiologically distinct strains. All six strains with a gyrA mutation contained wild-type rrs sequence.

Conclusions: Although fluoroquinolones are generally not used for chemotherapy of TB and drug susceptibility testing for second-line drugs is not carried out in Kuwait, four of 55 (7\%) individual patient MDR-TB strains contained mutations in gyrA gene. The data advocate routine drug susceptibility testing for this important second-line drug for proper management of MDR-TB in Kuwait. Lack of mutations in 3'-end of rrs gene that confer resistance to injectable agents reduce the likelihood of occurrence of XDR-TB, at present, in Kuwait.
\end{abstract}

Keywords: M. tuberculosis Fluoroquinolone resistance, gyrA mutations, Kuwait

* Correspondence: suhail_ah@hsc.edu.kw

Department of Microbiology, Faculty of Medicine, Kuwait University, Kuwait 


\section{Background}

Tuberculosis (TB), causing nearly 9 million active disease cases and two million deaths worldwide every year, is a major public health issue [1]. Increasing resistance of Mycobacterium tuberculosis strains to most-effective (first-line) anti-TB drugs and strong association of human immunodeficiency virus (HIV) pandemic with active TB disease are the two major contributors to the current global burden of TB [1-3]. Incomplete/improper treatment of TB patients leads to evolution of drugresistant $M$. tuberculosis strains due to chromosomal mutations in genes encoding drug targets [4]. Sequential accumulation of mutations in target genes generate multidrug-resistant (resistant at least to rifampicin and isoniazid) M. tuberculosis (MDR-TB) and extensively drugresistant (additionally resistant to fluoroquinolones and an injectable anti-TB agent such as kanamycin, amikacin or capreomycin) M. tuberculosis (XDR-TB) strains $[4,5]$. While proper treatment of drug-susceptible TB has $\geq 95 \%$ cure rate, effective treatment of MDR-TB is difficult in developing countries as it is heavily dependent on rapid diagnosis, supervised aggressive therapy with several (5-6) expensive, toxic and less efficacious (second-line) drugs for 18-24 months [4-7]. Inclusion of a fluoroquinolone and an injectable agent in multidrug treatment regimens have a more favorable outcome in the treatment of MDR-TB [4-8]. Treatment of XDR-TB is far more difficult even in developed countries while in developing countries, XDR-TB is virtually an untreatable disease [8-10]. The prognosis of XDR-TB in HIV-coinfected TB patients is extremely poor, with fatality rates varying from $\sim 30 \%$ in developed countries to nearly $100 \%$ in developing countries [8-11]. Hence, all efforts should be made to successfully cure the existing MDRTB cases to avoid the emergence of XDR-TB $[4,6,8,12]$.

Fluoroquinolones (FQs), particularly new generation compounds (such as moxifloxacin and gatifloxacin) and injectable aminoglycosides (kanamycin and amikacin) and cyclic peptide (capreomycin) have excellent bactericidal activity against $M$. tuberculosis and are crucial for proper management of MDR-TB patients $[4,8,12,13]$. Widespread emergence of MDR-TB has accelerated development of rapid drug susceptibility testing (DST) procedures for these important second-line drugs to help design effective treatment strategies. The cellular target of FQs in $M$. tuberculosis is DNA gyrase, a type II topoisomerase consisting of two A and two B subunits encoded by gyrA and $\operatorname{gyr} B$ genes, respectively [14]. Mutations in a small region of $g y r A$, called quinolone resistance-determining region (QRDR) and, less frequently, in $\operatorname{gyr} B$ are the primary mechanism of FQ resistance in M. tuberculosis [14-16]. Analysis of QRDR alone by genotypic tests has been suggested as sufficient for rapid identification of vast majority of FQ-resistant $M$. tuberculosis strains as additional targeting of gyrB did not enhance the sensitivity significantly $[16,17]$. The molecular basis of resistance of $M$. tuberculosis strains to injectable agents such as aminoglycosides (kanamycin and amikacin) and cyclic peptide (capreomycin) has also been determined $[4,18,19]$. Nearly $90 \%$ to $95 \%$ of $M$. tuberculosis strains resistant to one or more of the injectable agents contain mutations near the 3'-end of rrs (16S rRNA) gene involving nucleotide positions A1401, C1402, and G1484 [4,17-19].

The rate of MDR-TB is quite low ( 1\%) and FQs and injectable agents are rarely used for chemotherapy of TB in Kuwait [20]. This study was carried out to detect the occurrence of gyrA mutations associated with fluoroquinolone resistance among MDR-TB strains. The 3'end of rrs gene was also sequenced among isolates containing gyrA mutations to detect the occurrence of XDR-TB in Kuwait.

\section{Methods}

\section{Bacterial isolates and susceptibility testing}

A total of $4926 \mathrm{M}$. tuberculosis strains were isolated from TB patients during the study period (January 2001 to June 2008) at National Tuberculosis Reference Laboratory in Kuwait. A total of 110 M. tuberculosis isolates were analyzed in this study. These included 85 MDR-TB strains isolated from 55 TB patients (representing all available MDR $M$. tuberculosis strains). Twenty-five drug-susceptible $M$. tuberculosis strains (isolated from 25 patients) were also included to ensure that fluoroquinolone resistance-conferring mutations in the gyrA gene are not found in pansusceptible $M$. tuberculosis strains. M. tuberculosis $\mathrm{H}_{37} \mathrm{Rv}$ was used as a control. Isolation and identification of $M$. tuberculosis isolates was performed by using MGIT 960 system (Becton Dickinson) and multiplex PCR as described previously $[20,21]$. The phenotypic DST against first-line drugs (isoniazid, rifampicin, ethambutol, and streptomycin) was carried out by BACTEC $460 \mathrm{~TB}$ system as described in detail previously [20].

\section{DNA extraction for molecular assays}

One ml of MGIT 960 culture of reference or clinical $M$. tuberculosis isolate was heated with $40 \mathrm{mg}$ Chelex-100 (Sigma-Aldrich) at $95^{\circ} \mathrm{C}$ for $20 \mathrm{~min}$ and then centrifuged at $12,000 \times \mathrm{g}$ for $15 \mathrm{~min}$ [22]. For a PCR, $2 \mu \mathrm{l}$ of supernatant was used as a source of DNA.

\section{Detection of mutations in QRDR of the gyrA gene by DNA sequencing}

The QRDR of the gyrA gene was amplified by touchdown PCR by using GYRAF (5' - CGCAGCTACATCGACTATGCGATG-3') and GYRAR (5'-GGGATGAA 
ATCGATGTCTCCTCG-3') as amplification primers and the reaction and thermal cycling conditions as described in detail previously [23]. The $400 \mathrm{bp}$ amplicons were purified by using PCR product purification kit (Qiagen) and sequenced by using cycle DNA sequencing kit (DTCS CEQ2000, Beckman-Coulter) and GYRAFS (5'-CGGGTGCTCTATGCAATGTTC-3') or GYRARS (5'- GGCTTCGGTGTACCTCATCGCC-3') as internal sequencing primer. Although PCR products were purified, internal primers were used for sequencing of PCR amplicons to avoid interference from trace amounts of primer dimers, if still present. The detailed methodology of DNA sequencing was same as described in detail previously [23]. Nucleotide and amino acid sequences of the amplified products were compared with corresponding sequences from susceptible strain M. tuberculosis $\mathrm{H}_{37} \mathrm{Rv}$ using BLAST. DNA sequencing of QRDR also revealed the polymorphism (S95 or T95) that occurs at gyrA codon 95 among $M$. tuberculosis strains [24].

\section{Fingerprinting of MDR-TB strains carrying gyrA mutation} Fingerprinting of MDR-TB strains carrying $g y r A$ mutation was carried out by direct DNA sequencing of hotspot, N-terminal and cluster II regions of rpoB gene, inhA-regulatory region and katG codon 315 to detect mutations conferring resistance to rifampicin and isoniazid, as described in detail elsewhere [25]. The genetic group [24] of the isolates was determined by detecting the polymorphism at gyrA codon 95 (described above) and at katG codon 463 (L463 + T95, Group I; R463 + T95, Group II and R463 + S95, Group III). The presence of R463/L463 at katG codon 463 was detected by PCR amplification with KATG463F and KATG463R primers followed by restriction digestion with $N c i$ I, to generate RFLP patterns, as described previously [26]. Further fingerprinting of the isolates was carried out by double-repetitive-element (DRE)-PCR and isolates yielding unique patters were classified as genotypically distinct strains [27].

\section{Detection of mutations at 3'-end of rrs gene by DNA sequencing}

The 3'-end of rrs (16S rRNA) gene that is mutated in nearly $90 \%$ to $95 \%$ of $M$. tuberculosis strains exhibiting resistance to injectable agents (kanamycin, amikacin and capreomycin) [17-19] was also amplified by touchdown PCR by using 16S3F (5'-GCGATGCCGCGAGGTTAAGCGAA-3') and 16S3R (5'-CCAACAGTGTGTT GGTGGCCAA-3') as amplification primers and the reaction and thermal cycling conditions described previously [23]. The $403 \mathrm{bp}$ amplicons were purified and sequenced by using 16S3FS (5'-ATCCTTAAAAGC CGGTCTCAGT-3') or 16S3RS (5'- CTCCTTAGAAA
GGAGGTGATCCA-3') as internal sequencing primer. Again, internal primers were used for sequencing to avoid interference from trace amounts of primer dimers, if still present along with of PCR amplicons of rrs gene. The detailed DNA sequencing protocol has been described in detail previously [23]. Nucleotide and amino acid sequences of the amplified products were again compared with corresponding sequences from susceptible strain $M$. tuberculosis $\mathrm{H}_{37} \mathrm{Rv}$ using BLAST.

\section{Nucleotide sequence accession numbers}

The DNA sequencing data reported in this study have been deposited in EMBL under accession numbers FR734170-FR734173.

\section{Results}

Phenotypic DST data for 85 MDR-TB strains showed that $21(25 \%)$ isolates were resistant to isoniazid and rifampicin only while seven (8\%), $23(27 \%)$ and $34(40 \%)$ isolates were additionally resistant to streptomycin, ethambutol and to streptomycin and ethambutol, respectively (Table 1). Of the 55 patients yielding MDRTB strains, only two were Kuwaiti nationals while the remaining 53 patients were expatriate workers or their family members. The countries of origin for the 53 expatriate patients included India $(\mathrm{n}=24)$, Philippines $(\mathrm{n}=10)$, Egypt $(\mathrm{n}=7)$, Bangladesh $(\mathrm{n}=2)$, Indonesia ( $\mathrm{n}$ $=2)$, Syria $(n=2)$, Iraq $(n=2)$, Ethiopia $(n=1)$, Nepal $(\mathrm{n}=1)$, Nigeria $(\mathrm{n}=1)$ and Pakistan $(\mathrm{n}=1)$. The date of arrival in Kuwait was not available for the expatriate patients. All M. tuberculosis isolates were recovered from HIV-seronegative adult TB patients between the ages of 21 to 65 years and were resistant to the indicated drugs on first isolation; however, information on prior treatment of expatriate patients with anti-TB drugs was not available. The $25 \mathrm{M}$. tuberculosis isolates were susceptible to all first-line drugs (pansusceptible strains).

Table 1 Resistance patterns and presence of fluoroquinolone resistance-associated gyrA mutations in 110 M. tuberculosis strains tested.

\begin{tabular}{ccc}
\hline $\begin{array}{c}\text { Resistance pattern of } \\
\text { M. tuberculosis isolate }{ }^{\text {a }}\end{array}$ & $\begin{array}{c}\text { No. of isolates } \\
\text { tested }\end{array}$ & $\begin{array}{c}\text { No. of isolates with } \\
\text { gyrA mutation }\end{array}$ \\
\hline None & 25 & 0 \\
H, R & 21 & 0 \\
H, R, S & 7 & 0 \\
H, R, E & 23 & $4^{*}$ \\
H, R, S, E & 34 & 2 \\
Total & 110 & 6 \\
\hline
\end{tabular}

${ }^{a} H$, isoniazid; $R$, rifampicin, $S$, streptomycin; $E$, ethambutol

*3 of $4 \mathrm{M}$. tuberculosis isolates were recovered from the same TB patient 
All 110 isolates were identified as M. tuberculosis by a multiplex PCR that yielded 2 DNA fragments of $\sim 473$ bp and $\sim 235$ bp derived from oxyR and $r p o B$ genes, respectively, as expected (data from seven selected MDR-TB strains are shown in Figure 1). The PCR amplification of QRDR of gyrA gene from reference strain M. tuberculosis $\mathrm{H}_{37} \mathrm{Rv}$ as well as from all 25 pansusceptible and 85 MDR $M$. tuberculosis strains yielded an expected amplicon of $\sim 400 \mathrm{bp}$. The DNA sequencing data of all 25 pansusceptible $M$. tuberculosis strains showed complete concordance with wild-type gyrA sequence from reference strain except for polymorphism (S95 or T95) at gyrA codon 95, which is not associated with fluoroquinolone resistance [24]. The DNA sequencing data from 85 MDR-TB strains showed nucleotide (and amino acid) changes in QRDR of the gyrA gene in six isolates while the remaining 79 strains contained wild-type sequences except for gyrA codon 95 (Table 1). Among the six MDR-TB strains, nucleotide changes were detected only at gyrA codon 94, with five strains containing GAC94GGC (D94G) mutation and one isolate containing GAC94GCC (D94A) mutation. Interestingly, FQ resistance in MDR-TB strains was associated with additional resistance to ethambutol. Thus, all MDR-TB strains with gyrA mutations were resistant to three or all four first-line drugs (Table 1). Three of six FQ-resistant strains were isolated from the same patient within a period of two months while the remaining three isolates were recovered from three separate TB

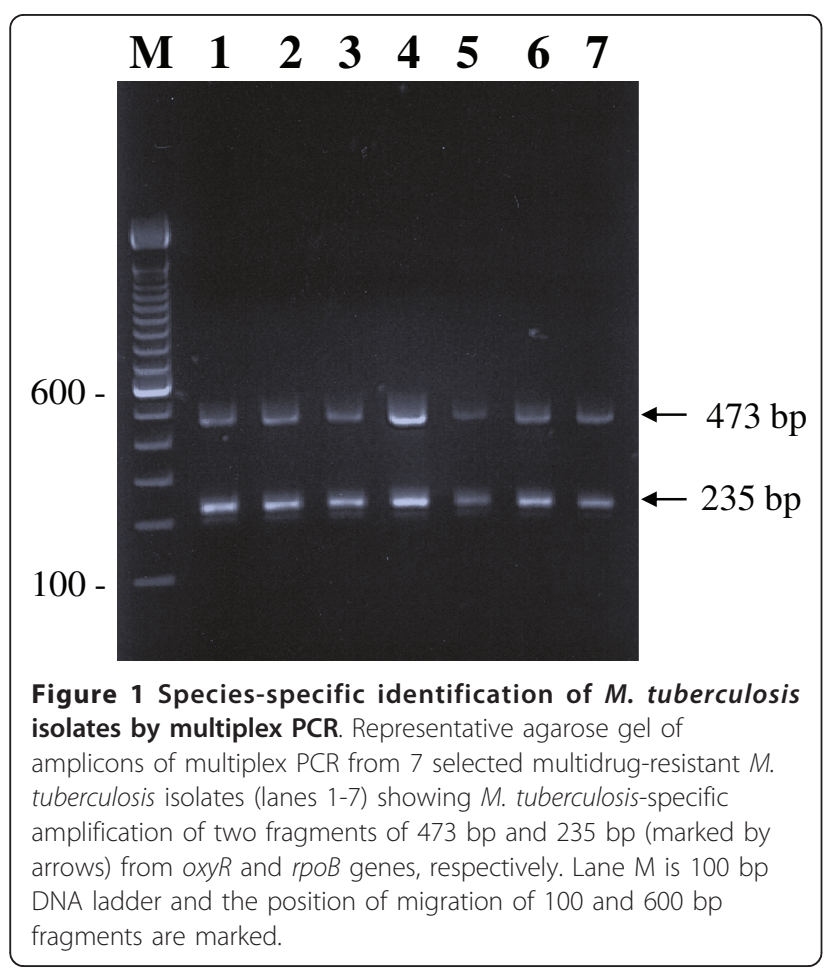

patients. Thus, four of 55 (7\%) individual patient MDRTB strains contained mutations in QRDR of the gyrA gene.

The year of isolation and results of fingerprinting studies for the four individual patient MDR-TB strains containing FQ resistance-associated gyrA mutations are presented in Table 2. The isolates were recovered over a four-year period (2004 to 2008) from male expatriate patients originating from India $(\mathrm{n}=3)$ and Bangladesh $(\mathrm{n}=1)$. Three patients developed pulmonary TB while the fourth patient had extrapulmonary form of the disease. Fingerprinting of the isolates based on specific mutations in hot-spot, $\mathrm{N}$-terminal and cluster II regions of rpoB, kat $G$ codon 315 and inhA-regulatory region and genetic group analyses showed that all four isolates were distinct strains (Table 2). All four isolates also yielded unique DRE-PCR patterns. The two repeat isolates recovered from one patient (no. 3) exhibited the same fingerprinting patterns as the first isolate from this patient (data not shown).

\section{Discussion}

Kuwait, an Arabian Gulf country with nearly 25 cases per 100, 000 population is a low TB incidence country [28]. However, it has a large expatriate population originating from TB endemic countries of South-Southeast Asia. Nearly 600 patients are diagnosed with mycobacterial infections every year. Nearly $95 \%$ of mycobacterial infections are caused by $M$. tuberculosis (TB) while the remaining $5 \%$ are caused by non-tuberculous mycobacteria [29]. The non-tuberculous mycobacterial infections are more common among Kuwaiti patients, however, $\sim 80 \%$ of TB patients are expatriate workers or their family members $[20,29]$. This is despite the fact that all expatriates are screened for TB and HIV on entry and are allowed to stay in Kuwait only if they exhibit no obvious signs (suspected lesions on chest radiograph) of active TB disease or previous exposure to active disease. Furthermore, all non-infectious TB cases are sent back (after anti-TB therapy of 4-8 weeks, if required) to their respective countries for further management [20]. While global proportion of MDR-TB has been estimated to be $\sim 5.3 \%$ among all cases [2,3], only $\sim 1 \%$ of $M$. tuberculosis isolates in Kuwait are MDR-TB strains and nearly all of these strains are isolated from expatriate patients [20]. The incidence of extrapulmonary TB in Kuwait is relatively high, accounting for $44 \%$ of all TB cases and majority of pulmonary TB patients have cavitary lesions in the upper lobes $[20,28]$. These findings together with the low incidence of TB in Kuwait are consistent with observations that majority of active TB disease cases in foreign-born persons occur as a result of reactivation of prior (latent) infection, usually within the first few years of their migration [30]. Previous fingerprinting studies 
Table 2 Patient's demographic data and fingerprinting data for the four individual patient multidrug-resistant $M$. tuberculosis strains containing a mutation in quinolone resistance-determining region (QRDR) of gyrA gene.

\begin{tabular}{|c|c|c|c|c|c|c|c|c|}
\hline \multirow{2}{*}{$\begin{array}{c}\text { Patient } \\
\text { no. }\end{array}$} & \multirow[t]{2}{*}{ Nationality } & \multirow{2}{*}{$\begin{array}{c}\text { Clinical } \\
\text { specimen }\end{array}$} & \multirow{2}{*}{$\begin{array}{l}\text { Year of } \\
\text { isolation }\end{array}$} & \multirow{2}{*}{$\begin{array}{c}\text { Resistance } \\
\text { pattern }^{\mathrm{a}}\end{array}$} & \multirow{2}{*}{$\begin{array}{c}\text { Mutation in QRDR } \\
\text { of gyrA gene }\end{array}$} & \multicolumn{3}{|c|}{ Fingerprinting data from analysis of $^{b}$} \\
\hline & & & & & & $r p o B^{\mathrm{C}}$ & inhA-RR ${ }^{\mathrm{d}}$ & Genetic group $^{\mathrm{e}}$ \\
\hline 1 & Indian & Pleural fluid & 2004 & $H, R, S, E$ & GAC94GGC/D94G & Wild-type $^{f}$ & WT & I \\
\hline 2 & Bangladeshi & Sputum & 2006 & $H, R, S, E$ & GAC94GCC/D94A & $H 526 \mathrm{Q}+\mathrm{D} 516 \mathrm{G}$ & WT & III \\
\hline $3^{*}$ & Indian & Sputum & 2006 & $H, R, E$ & GAC94GGC/D94G & I572F & WT & । \\
\hline 4 & Indian & Sputum & 2008 & $\mathrm{H}, \mathrm{R}, \mathrm{E}$ & GAC94GGC/D94G & S531L & $-17 \mathrm{G} \rightarrow \top$ & 1 \\
\hline
\end{tabular}

${ }^{a} H$, isoniazid; $R$, rifampicin, $S$, streptomycin; $E$, ethambutol.

${ }^{b}$ All four were male patients and all individual patient isolates contained AGC315ACC mutation in katG codon 315 and yielded unique patterns in DRE-PCR.

${ }^{C}$ Three (hot-spot, N-terminal and cluster II) regions of $r p o B$ gene were sequenced to detect rifampicin resistance-conferring mutations and codon numbering system of $r p o B$ gene from Escherichia coli is used [4].

$\mathrm{d}$ InhA-RR, inhA regulatory region sequencing data.

'Genetic groups were assigned based on polymorphisms at katG codon 463 and gyrA codon 95(L463/T95, Group I; R463/S95, Group III)

${ }^{\mathrm{f}}$ This isolate either contained a mutation in other regions of the $r p o B$ gene or in other genes involved in rifampicin resistance [25].

*Two repeat isolates recovered from this patient exhibited identical patterns.

have also shown that vast majority of MDR-TB cases among expatriate patients in Kuwait have unique patterns [31,32]. Since fingerprinting patterns are highly variable in countries that have a low incidence of active TB disease and immigrants originating from high incidence countries [33], these observations also support reactivation of previously acquired infection as the major mechanism for active TB disease in most patients in Kuwait.

The FQs play an important role in the treatment of MDR-TB since their inclusion in therapy regimens improves treatment outcome. Resistance of MDR-TB strains to FQs is associated with poor treatment outcome and is also one of two key defining conditions of XDR-TB $[4,7-10,12]$. Thus, there is a pressing need for rapid DST of MDR-TB strains against FQs to improve clinical management. However, like many other countries [14], routine DST for second-line drugs has not been instituted in the National Tuberculosis Control Program in Kuwait, mainly due to low rate of MDR-TB [20]. Before planning for routine phenotypic and/or genotypic DST for FQs and other second-line drugs in Kuwait, this study was carried out to detect the occurrence of $g y r A$ mutations associated with FQ resistance among MDR-TB strains.

Our data showed that four of 55 (7\%) individual patient MDR-TB isolates in Kuwait contained mutations in QRDR of the gyrA gene. Interestingly, all MDR-TB strains with a gyrA mutation were additionally resistant to ethambutol with/without additional resistance to streptomycin. Previous studies have also noted an association of FQ resistance in $M$. tuberculosis strains with additional resistance to several first-line drugs $[15,17]$. All four individual patient isolates contained a mutation at gyrA codon 94 which was also the most frequently mutated codon among FQ-resistant $M$. tuberculosis strains in several previous studies $[15,17,34-37]$. Three of four individual patient isolates with gyrA mutation contained D94G mutation while the fourth isolate contained D94A mutation. Studies from Germany, Taiwan and Russia have also reported that D94G is the most common mutation observed in FQ-resistant $M$. tuberculosis strains $[15,17,35]$ while FQ-resistant strains from Japan contained D94A as the most frequent mutation [37]. Although phenotypic drug susceptibility testing for FQs was not performed in this study, the level of resistance in FQ-resistant $M$. tuberculosis strains could be inferred based on the nature and kind of $\operatorname{gyr} A$ mutations detected. Previous studies have shown that $M$. tuberculosis strains containing D94G mutation in the gyrA gene exhibit minimum inhibitory concentration (MIC) of $\geq 4$ $\mu \mathrm{g} / \mathrm{ml}$ while those with D94A mutation have MIC of 2 $\mu \mathrm{g} / \mathrm{ml}$ for levofloxacin $[37,38]$. Based on these observations, it may be inferred that three of four FQ-resistant $M$. tuberculosis strains from Kuwait were highly resistant to fluoroquinolones.

Three of four patients infected with M. tuberculosis strains containing gyrA mutation originated from India while the fourth patient was from Bangladesh. Since FQs are frequently used for other bacterial infections, it is probable that many expatriate patients were exposed to these agents in their respective native countries. Recent data have shown that $24 \%$ and $9 \%$ of MDR-TB strains from India and Bangladesh, respectively, are resistant to FQs [5]. The MDR-TB strains containing gyrA mutations were cultured at the time of clinical diagnosis before anti-TB treatment was initiated (primary resistance) and not during treatment during a fiveyear period and were genotypically distinct strains. These observations together with the low incidence of active TB disease and low rates of active transmission of infection in Kuwait [20,28-30] imply that the four expatriate patients did not acquire the infection recently but were most likely latently infected with FQ-resistant 
strains. A limitation of the present study is that phenotypic DST for FQs was not performed. Previous studies from several geographical locations have shown that nearly $70 \%$ to $100 \%$ of FQ-resistant $M$. tuberculosis strains contain mutations in QRDR of the gyrA gene while FQ resistance in other isolates is associated with mutations outside of QRDR of $\operatorname{gyr} A$ gene or due to $\operatorname{gyr} B$ gene mutations [15,17,34-38]. It is, therefore, probable that phenotypic DST may have identified few (one or two) additional FQ-resistant MDR-TB strains in Kuwait. Thus, phenotypic DST should be performed as an important preliminary tool to determine the real prevalence of FQ resistance in Kuwait.

The absence of mutations in 3'-end of rrs gene, which confer resistance to kanamycin, amikacin or capreomycin [17-19], among the six FQ-resistant MDR-TB strains is reassuring as it decreases the possibility of the presence of XDR-TB in Kuwait. This is most likely due to very infrequent use of these injectable agents for the treatment of TB in Kuwait.

\section{Conclusions}

The detection of fluoroquinolone resistance-associated mutations in gyrA gene in four of 55 (7\%) individual patient MDR-TB strains strongly suggest the need for routine drug susceptibility testing for this important second-line drug for proper management of MDR-TB in Kuwait. However, lack of mutations in 3'-end of rrs gene that confer resistance to injectable agents is encouraging and rules out, at least for now, the presence of XDR-TB in Kuwait.

\section{Acknowledgements \\ This study was supported by Research Administration grant YM03/06 and College of Graduate Studies, Kuwait University.}

\section{Authors' contributions}

SA and EM designed the study and NMA carried out the experiments. All authors contributed in manuscript writing. All of the authors have read and approved the final manuscript.

\section{Competing interests}

The authors declare that they have no competing interests.

Received: 17 December 2010 Accepted: 14 April 2011

Published: 14 April 2011

\section{References}

1. World Health Organization: Global tuberculosis control: WHO report 2010. WHO/HTM/TB/2010.7. Geneva, Switzerland: WHO; 2010.

2. World Health Organization: Anti-tuberculosis drug resistance in the world: fourth Global report. WHO/HTM/TB/2008.394. Geneva, Switzerland: WHO; 2008.

3. Wright A, Zignol M, Van Deun A, Falzon D, Gerdes SR, Feldman K, Hoffner S, Drobniewski F, Barrera L, van Soolingen D, Boulabhal F, Paramasivan CN, Kam KM, Mitarai S, Nunn P, Raviglione M, for the Global Project on Anti-Tuberculosis Drug Resistance Surveillance: Epidemiology of antituberculosis drug resistance 2002-2007: an updated analysis of the Global Project on Anti-Tuberculosis Drug Resistance Surveillance. Lancet 2009, 373:1861-1873
4. Ahmad S, Mokaddas E: Recent advances in the diagnosis and treatment of multidrug-resistant tuberculosis. Resp Med 2009, 103:1777-1790.

5. World Health Organization: Multidrug and extensively drug-resistant TB (M/XDR-TB): 2010 global report on surveillance and response. WHO/ HTM/TB/2010.3. Geneva, Switzerland: WHO; 2010.

6. Mitnick CD, Castro KG, Harrington M, Sacks LV, Burman W: Randomized trials to optimize treatment of multidrug-resistant tuberculosis. PLOS Med 2007, 4:article no. e292.

7. Orenstein EW, Basu S, Shah NS, Andrews JR, Friedland GH, Moll AP, Gandhi NR, Galvani AP: Treatment outcomes among patients with multidrug-resistant tuberculosis: systematic review and meta-analysis. Lancet Infect Dis 2009, 9:153-161.

8. Ahmad $\mathrm{S}$, Mokaddas E: New challenges in the treatment of tuberculosis in the $21^{\text {st }}$ century. Treat Strat Resp 2011, 1:100-110.

9. Mitnick CD, Shin SS, Seung GY, Rich ML, Atwood SS, Furin JJ, Fitzmaurice GM, Alcantara Viru FA, Appleton SC, Bayona JN, Bonilla CA, Chalco K, Choi S, Franke MF, Fraser HS, Guerra D, Hurtado RM, Jazayeri D, Joseph K, Llaro K, Mestanza L, Mukherjee JS, Muñoz M, Palacios E, Sanchez E, Sloutsky A, Becerra MC: Comprehensive treatment of extensively drug-resistant tuberculosis. N Engl J Med 2008, 359:563-574.

10. Jacobson KR, Tierney DB, Jeon CY, Mitnick CD, Murray MB: Treatment outcomes among patients with extensively drug-resistant tuberculosis: systematic review and meta-analysis. Clin Infect Dis 2010, 51:6-14.

11. Gandhi NR, Moll A, Sturn AW, Pawinski R, Govender T, Lalloo U, Zeller K, Andrews J, Friedland G: Extensively drug-resistant tuberculosis as a cause of death in patients co-infected with tuberculosis and HIV in a rural area of South Africa. Lancet 2006, 368:1554-1556.

12. Kliman K, Altraja A: Predictors of poor treatment outcome in multi and extensively drug-resistant pulmonary TB. Eur Respir J 2009, 33:1085-1094.

13. American Thoracic Society, CDC, Infectious Disease Society of America: Treatment of tuberculosis. Morb Mort Weekly Rep 2003, 52:1-77.

14. Ginsburg AS, Grosset JH, Bishai WR: Fluoroquinolones, tuberculosis, and resistance. Lancet Infect Dis 2003, 3:432-442.

15. Wang JY, Lee LN, Lai HC, Wang SK, Jan IS, Yu CJ, Hsueh PR, Yang PC: Fluoroquinolones resistance in Mycobacterium tuberculosis isolates: associated genetic mutations and relationship to antimicrobial exposure. J Antimicrob Chemother 2007, 59:860-865.

16. Chang KC, Yew WW, Chan RCY: Rapid assays for fluoroquinolone resistance in Mycobacterium tuberculosis: a systematic review and metaanalysis. J Antimicrob Chemother 2010, 65:1551-1561.

17. Hillemann D, Rusch-Gerdes S, Richter E: Feasibility of the GenoType MTBDRs/ assay for fluoroquinolone, amikacin-capreomycin and ethambutol resistance testing of Mycobacterium tuberculosis strains and clinical specimens. J Clin Microbiol 2009, 47:1767-1772.

18. Maus CE, Pilkaytis BB, Shinnick TM: Molecular analysis of cross-resistance to capreomycin, kanamycin, amikacin and viomycin in Mycobacterium tuberculosis. Antimicrob Agents Chemother 2005, 49:3192-3197.

19. Jugheli L, Bzekalava N, de Rijk P, Fissette K, Portaels F, Rigouts L: High level cross-resistance between kanamycin, amikacin and capreomycin among Mycobacterium tuberculosis isolates from Georgia and a close relation with mutations in the rrs gene. Antimicrob Agents Chemother 2009, 53:5064-5068

20. Mokaddas E, Ahmad S, Samir I: Secular trends in susceptibility patterns of Mycobacterium tuberculosis isolates in Kuwait, 1996-2005. Int J Tuberc Lung Dis 2008, 12:319-325.

21. Mokaddas E, Ahmad S: Development and evaluation of a multiplex PCR for rapid detection and differentiation of Mycobacterium tuberculosis complex members from non-tuberculous mycobacteria. Jpn J Infect Dis 2007, 60:140-144.

22. Jaber AA, Ahmad S, Mokaddas E: Minor contribution of mutations at iniA codon 501 and embC-embA intergenic region in ethambutol-resistant clinical Mycobacterium tuberculosis isolates in Kuwait. Ann Clin Microbiol Antimicrob 2009, 8:2.

23. Ahmad S, Mokaddas E, Jaber AA: Rapid detection of ethambutol-resistant Mycobacterium tuberculosis strains by PCR-RFLP targeting embB codons 306 and 497 and iniA codon 501 mutations. Mol Cell Probes 2004, 18:299-306.

24. Sreevatsan S, Pan X, Stockbauer KE, Connell ND, Kreiswirth BN, Whittam TS, Musser JM: Restricted structural gene polymorphism in the Mycobacterium tuberculosis complex indicates evolutionarily recent global dissemination. Proc Natl Acad Sci USA 1997, 94:9869-9874. 
25. Al-Mutairi N, Ahmad S, Mokaddas E: Performance comparison of four methods for rapid detection of multidrug-resistant Mycobacterium tuberculosis strains. Int J Tuberc Lung Dis 2011, 15:110-115.

26. Ahmad S, Jaber AA, Mokaddas E: Frequency of embB codon 306 mutations in ethambutol-susceptible and -resistant clinical Mycobacterium tuberculosis isolates in Kuwait. Tuberculosis 2007, 87:123-129.

27. Mokaddas E, Ahmad S, Abal AT: Molecular fingerprinting of isoniazidresistant Mycobacterium tuberculosis isolates from Chest Diseases Hospital in Kuwait. Microbiol Immunol 2002, 46:767-771.

28. Behbehani N, Abal A, Al-Shami A, Enarson DA: Epidemiology of tuberculosis in Kuwait from 1965 to 1999. Int J Tuberc Lung Dis 2002, 6:465-469.

29. Mokaddas E, Ahmad S: Species spectrum of nontuberculous mycobacteria isolated from clinical specimens in Kuwait. Curr Microbiol 2008, 56:413-417.

30. Ahmad S: New approaches in the diagnosis and treatment of latent tuberculosis infection. Resp Res 2010, 11:169.

31. Ahmad S, Mokaddas E, Fares E: Characterization of rpoB mutations in rifampin-resistant clinical Mycobacterium tuberculosis isolates from Kuwait and Dubai. Diagn Microbiol Infect Dis 2002, 44:245-252.

32. Ahmad S, Mokaddas E: The occurrence of rare rpoB mutations in rifampicin-resistant Mycobacterium tuberculosis isolates from Kuwait. Int $\rfloor$ Antimicrob Agents 2005, 26:205-212.

33. Van Soolingen D: Molecular epidemiology of tuberculosis and other mycobacterial infections: main methodologies and achievements. J Intern Med 2001, 249:1-26.

34. Shi R, Zhang J, Li C, Kazumi Y, Sugawara I: Emergence of ofloxacin resistance in Mycobacterium tuberculosis clinical isolates from China as determined by gyrA mutation analysis using denaturing high-pressure liquid chromatography and DNA sequencing. I Clin Microbiol 2006, 44:4566-4568.

35. Mokrousov I, Otten T, Manicheva O, Potapova Y, Vishnevsky B, Narvskaya O, Rastogi N: Molecular characterization of ofloxacin-resistant Mycobacterium tuberculosis strains from Russia. Antimicrob Agents Chemother 2008, 52:2937-2939.

36. van Doorn HR, An DD, de Jong MD, Lan NTN, Hoa DV, Quy HT, Chau NW, Duy PM, Tho DQ, Chinh NT, Farrar JJ, Caws M: Fluoroquinolone resistance detection in Mycobacterium tuberculosis with locked nucleic acid probe real-time PCR. Int J Tuberc Lung Dis 2008, 12:736-742.

37. Ando H, Mitarai $S$, Kondo Y, Suetake T, Kato S, Mori T, Kirikae T: Evaluation of a line probe assay for the rapid detection of gyrA mutations associated with fluoroquinolone resistance in multidrug-resistant Mycobacterium tuberculosis. J Med Microbiol 2011, 60:184-188.

38. Giannoni F, lona E, Sementilli F, Brunori L, Pardini M, Migliori GB, Orefici G Fattorini $L$ : Evaluation of a line probe assay for the rapid identification of gyrA mutations in Mycobacterium tuberculosis. Antimicrob Agents Chemother 2005, 49:2928-2933.

doi:10.1186/1756-0500-4-123

Cite this article as: Al-Mutairi et al:: First report of molecular detection of fluoroquinolone resistance-associated gyrA mutations in multidrugresistant clinical Mycobacterium tuberculosis isolates in Kuwait. BMC Research Notes 2011 4:123.

\section{Submit your next manuscript to BioMed Central and take full advantage of:}

- Convenient online submission

- Thorough peer review

- No space constraints or color figure charges

- Immediate publication on acceptance

- Inclusion in PubMed, CAS, Scopus and Google Scholar

- Research which is freely available for redistribution

Submit your manuscript at www.biomedcentral.com/submit
Biomed Central 